\title{
Systemic Lupus Erythematosus from Lupus Enteritis
}

\author{
Junichi Kawai, Kazuki Kakimoto, Hirota Miyazaki, Yuki Hirata, Ken Kawakami, Kazuhide Higuchi
}

$2^{\text {nd }}$ Department of Internal Medicine, Osaka Medical College, Takatsuki, Osaka, Japan

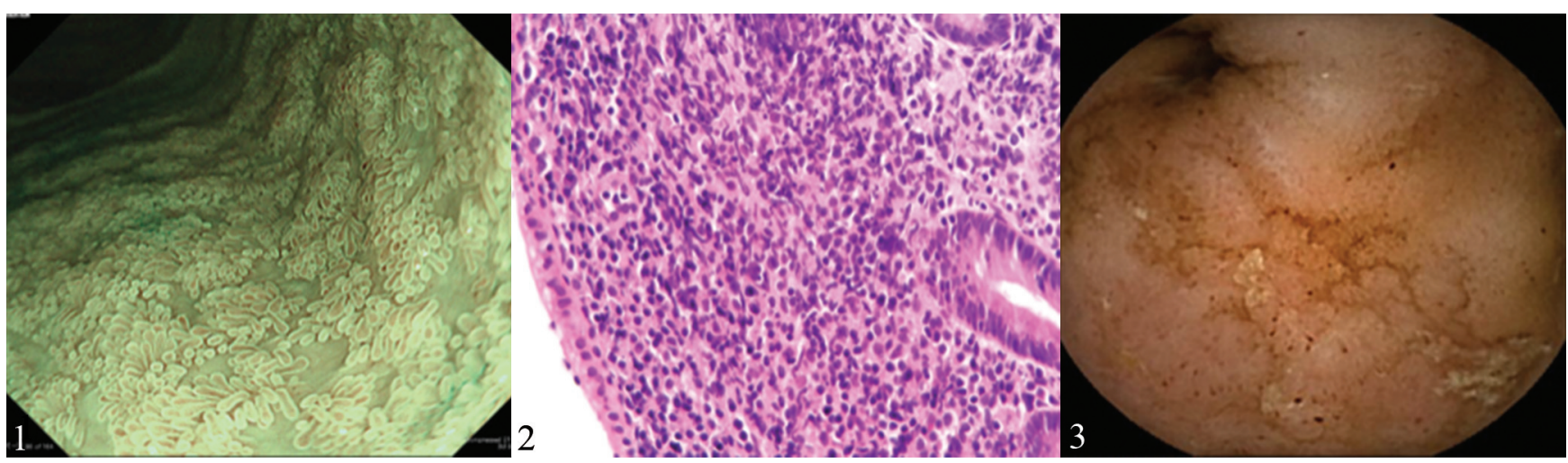

A 24-year old woman was admitted for persistent abdominal pain, watery diarrhea, and vomiting for the last 3 months. Laboratory tests indicated mild inflammation with a C-reactive protein level of $0.72 \mathrm{mg} / \mathrm{dL}$ and a white blood cell count of $7290 /$ $\mathrm{mm} 3$. No pathogenic bacteria were detected on a stool culture. Computed tomography revealed extensive thickening of the small intestine and ascites. Endoscopy showed a normal mucosa of the large bowel, except for some areas of the terminal ileum where narrow-band imaging evidenced the disappearance of the villi (Fig. 1). The histological examination showed severe lymphocyte infiltration into the lamina propria, but no definitive vasculitis (Fig 2, H\&E, ×200). Capsule endoscopy revealed extensive petechiae throughout the upper jejunum and pelvic ileum, and disappearance of the villi (Fig 3). One month after admission, the patient developed a butterfly rash and arthritis. Laboratory tests indicated a 640-fold increase in the level of antinuclear antibodies and an elevation of the anti-double stranded deoxyribonucleic acid antibody levels (35.2 IU/L normal <12.0 IU/L). The patient was subsequently diagnosed with systemic lupus erythematous (SLE) and lupus enteritis. Prednisolone (60 mg daily) and tacrolimus (2 mg daily) were initiated with clinical remission.

The incidence of lupus enteritis in SLE is reported to be 8-27\% [1]. Lupus enteritis presents as edematous submucosa with diffuse inflammatory infiltration of mononuclear cells and vasculitis extending from the submucosal layer to the subserosa, which incurs difficult detection in intestinal biopsies [2]. The mean duration from SLE diagnosis to lupus enteritis onset is generally 5 years [3]. Only $1.3 \%$ of patients develop lupus enteritis at SLE onset [3]. Cases like our patient, in whom the only symptom at SLE onset was lupus enteritis, are extremely rare. It is necessary to consider the possibility of lupus enteritis even in the absence of typical SLE symptoms. Mucosal edema has been initially reported as a small bowel lesion depicted at endoscopy. However, with recent advances in small intestinal endoscopy, erosions, ulcers and scars have been associated with lupus enteritis [4].

Corresponding author: Kazuki Kakimoto, in2098@osaka-med.ac.jp

Conflicts of interest: None to declare.

\section{REFERENCES}

1. Sultan SM, Ioannou Y, Isenberg DA. A review of gastrointestinal manifestations of systemic lupus erythematosus. Rheumatology (Oxford) 1999;38:917-932. doi:10.1093/rheumatology/38.10.917

2. Tian XP, Zhang X. Gastrointestinal involvement in systemic lupus erythematosus: insight into pathogenesis, diagnosis and treatment. World J Gastroenterol 2010;16:2971-9727. doi:10.3748/wjg.v16.i24.2971

3. Janssens P, Arnaud L, Galicier L, et al. Lupus enteritis: from clinical findings to therapeutic management. Orphanet J Rare Dis 2013;8:67. doi:10.1186/1750-1172-8-67

4. Endo H, Kondo Y, Kawagoe K, et al. Lupus enteritis detected by capsule endoscopy. Intern Med 2007;46:1621-1622. doi:10.2169/ internalmedicine.46.0137 